Thorax, 1978, 33, 493-499

\title{
Pulmonary and systemic aneurysms in a case of widespread arteritis
}

\author{
J P R HARTLEY, J S DINNEN, AND A SEATON ${ }^{1}$
}

From the Asthma Research Unit, Sully Hospital, Penarth, and the Department of Pathology, ,.' University Hospital of Wales, Cardiff, S Glamorgan, Wales

\begin{abstract}
Hartley, J P R, Dinnen, J S, and Seaton, A (1978). Thorax, 33, 493-499. Pulmonary and systemic aneurysms in a case of widespread arteritis. A patient in whom asthma preceded the development of multiple pulmonary and systemic artery aneurisms died after dissection of the aorta. At necropsy he was found to have widespread arteritis of unknown aetiology affecting many large and medium-sized pulmonary and systemic vessels as well as a few microscopic ones. Endarteritis obliterans was present in some of the vasa vasorum. The clinical and histopathological findings are discussed in relationship to other known causes of arteritis, and it is concluded that this condition has not previously been described.
\end{abstract}

Pulmonary artery aneurysms are conventionally divided into two groups. Those occurring in the large extrapulmonary vessels are most commonly associated with congenital cardiac abnormalities (Boyd and McGavack, 1939; Deterling and Clagett, 1947), while those occurring in the smaller intrapulmonary divisions are usually mycotic (Charlton and DuPlessis, 1961). All pulmonary artery aneurysms are extremely rare, and it is even less common to find pulmonary and systemic arterial aneurysms in the same patient. We report a case in which this occurred, secondary to widespread arteritis, and in which the pulmonary component raised diagnostic problems.

\section{Case report}

The patient, a white engineer, developed asthma in 1970 at the age of 50 . He was admitted to hospital three times with severe attacks of asthma during that year. On each occasion he responded well to corticosteroid treatment, and he was treated with intermittent courses of prednisone and with bronchodilators as an outpatient between these episodes. Typical asthmatic stigmata were present in the sputum (Sanerkin and Evans, 1965), but there was never a peripheral blood eosinophilia. Skin tests to common allergens and precipitins to Aspergillus fumigatus were negative. The ESR (Westergren) was always less than $12 \mathrm{~mm}$ 1Present address: Institute of Occupational Medicine, 8 Roxburgh
Place, Edinburgh, UK. in the first hour, and chest radiographs were normal. During the next four years the patient was admitted to hospital only twice, though by 1973 he needed continuous prednisone treatment.

In March 1975 he was admitted to hospital with a three-week history of vertigo, recurrent vomiting, and intermittent loss of vision in the left eye. On questioning he described symptoms suggestive of Raynaud's phenomenon in his right hand, angina pectoris, and intermittent claudication, which had all developed over the previous two months. Physical examination showed bilateral femoral artery bruits, a bruit over the left subclavian artery, and a right subclavian aneurysm. At this time his ESR was $66 \mathrm{~mm}$ in the first hour, ANF was negative, but the DAT for rheumatoid factor was positive to a titre of $1 / 128$. He had a peripheral blood eosinophil count of $790 \times 10^{6} / 1$, the first time this had been raised since he developed asthma. His brain-stem symptoms were thought to be due to a subclavian-steal syndrome, associated with a systemic arteritis, but within a week all his symptoms disappeared without specific treatment.

The chest radiograph now showed apparent right hilar enlargement (fig 1). He was a nonsmoker, and the results of sputum examination for malignant cells were persistently negative. Fibreoptic bronchoscopy showed no intrabronchial lesion, and a Kveim test result was negative. Tomography was interpreted as showing rightsided hilar and paratracheal lymph node enlarge- 


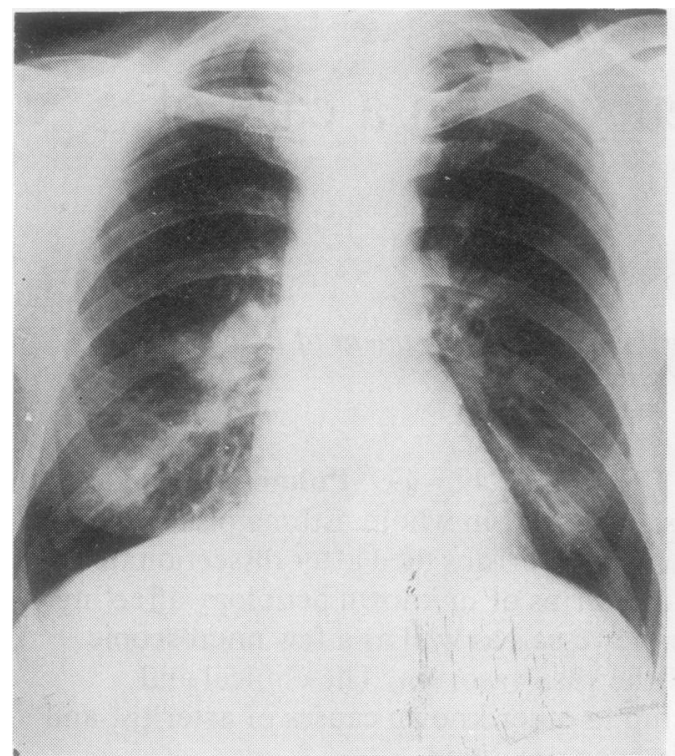

Fig 1 Chest radiograph in March 1975, when right hilar enlargement was first noted.

ment, and a lymphangiogram was reported to show abnormal iliac and para-aortic nodes compatible with malignant lymphoma. A laparotomy and splenectomy were performed in May 1975 for diagnosis and staging. The findings were unhelpful. No enlarged para-aortic nodes were found, and the liver, spleen, and three mesenteric lymph nodes showed no evidence of lymphoma.

The patient was by now asymptomatic, and he was observed as an outpatient. Because the right hilar shadow had increased in size he was readmitted in November 1975, and a thoracotomy was performed. This showed no lymph node enlargement; instead, multiple pulmonary and systemic aneurysms were found. The pulmonary artery was dilated and aneurysmal in several branches, particularly those to the right lower lobe. Large, lobulated aneurysms of the innominate and right subclavian arteries were present, and the right internal mammary artery was thrombosed. A segment of this vessel was removed, and the chest wall closed. Histological examination showed that the media and internal elastic lamina were completely destroyed and replaced by fibrous tissue. There was a heavy infiltrate of plasma cells and lymphocytes in the outer half of the vessel wall.

Subsequent pulmonary angiography (fig 2) showed the extent of pulmonary vascular involvement. The main pulmonary artery trunk and proximal pulmonary arteries were radiologically

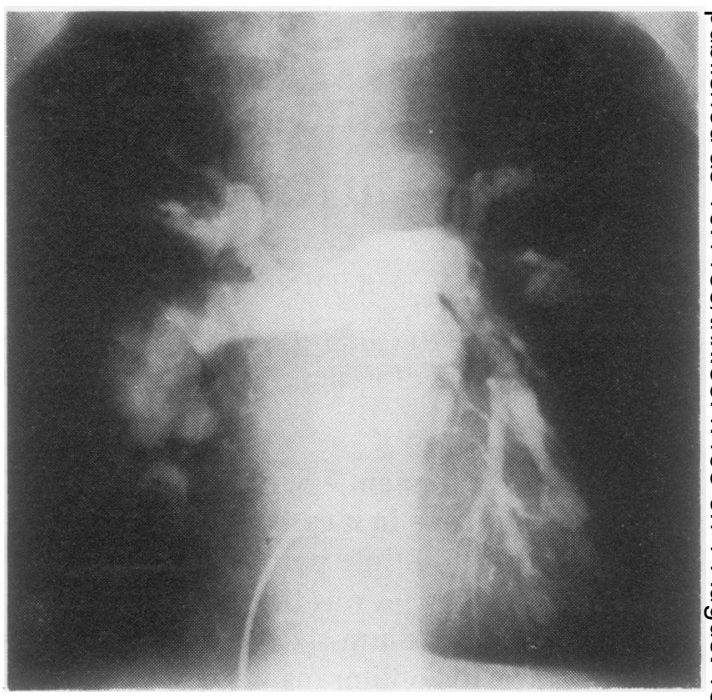

Fig 2 Pulmonary angiogram showing multiple aneurysms in medium-sized arteries. Pulmonary trunk and proximal pulmonary arteries are normal.

normal, but beyond this, multiple aneurysms were气 present in the medium-sized pulmonary artery branches bilaterally. The aorta appeared normal, $\overline{\overrightarrow{0}}$ but large aneurysms of the aortic arch branches were seen. Intracardiac and pulmonary artery pressures were normal. In January 1976 the patient remained well. He was normotensive and had normal renal function. Tests for rheumatoid fac-o tor and ANF were negative, as were serological tests for syphilis (WR, Kahn, and RPCFT), and serum immunoglobulin concentrations were nor-i mal (IgG $14.0 \mathrm{~g} / 1, \operatorname{IgA} 2.9 \mathrm{~g} / 1$ and IgM $1.1 \mathrm{~g} / 1)$. At? no time was the skin affected. Treatment with prednisone, $20 \mathrm{mg}$ daily, and azathioprine, $150 \mathrm{mg}$ daily, was started. The patient died suddenly in? April 1976, 13 months after his initial presentation with arteritic symptoms.

NECROPSY

The cause of death was a haemopericardium resulting from rupture of a localised dissecting aneurysm of the aorta. One centimetre above the aortic valve there was a $2.5 \mathrm{~cm}$ transverse tear in the intima. The underlying dissection in the media measured $2.5 \times 2 \mathrm{~cm}$, and there was a small perforation into the pericardial cavity. The thoracic aorta was not dilated but showed a moderate $\frac{?}{?}$ number of atheromatous plaques, and the inter- 0 vening intima was grey and slightly irregular. The abdominal aorta and common iliac arteries showedo confluent atheroma. There was widespreado 
aneurysm formation in other systemic arteries. The innominate artery was dilated, and both subclavian arteries had fusiform aneurysms up to $4 \mathrm{~cm}$ in diameter. Aneurysms $2 \mathrm{~cm}$ in diameter and partly filled by thrombus were present on both left and right coronary arteries, close to their origins (fig 3). Smaller aneurysms were present on the left common carotid, superior mesenteric, and left renal arteries. In the lungs the pulmonary artery branches to the anterior segments of both upper lobes, the right middle lobe, apical segment of right lower lobe, and the anterior basal segment of the left lower lobe all had aneurysms of 1-2 cm in diameter. No abnormality was found in the peripheral veins of the legs or the dural sinuses.

\section{Histology}

There was a widespread arteritis affecting mainly large systemic and pulmonary vessels. The thoracic and abdominal aorta showed infiltration of the outer layers of the media by prominent vessels, which were surrounded by plasma cells, lymphocytes, and occasional macrophages and eosinophils. In many places the infiltrate extended right through the media, and the elastic tissue was completely replaced by collagen (fig 4). The ad- ventitia had a similar inflammatory infiltrate, and there was endarteritis obliterans of some of the vasa vasorum. The intima showed fibrous thickening with foci of lymphocytes and with atheromatous deposits. There were no giant cells or granulomas. No spirochaetes were shown by Levaditi's method, and the aorta showed no evidence of cystic medionecrosis when stained with alcian blue. The dissection itself lay in the inflamed outer third of the media in the ascending aorta.

The systemic and pulmonary aneurysms (fig 5), and vessels such as the splenic and cornmon iliac arteries, showed complete destruction of the media and replacement by fibrous tissue, with a similar inflammatory infiltrate. Medial destruction in these vessels was more severe than in the aorta, representing the end stage of the arteritic process. The pulmonary trunk and main pulmonary arteries showed slight intimal thickening with occasional chronic inflammatory cells only, and the microscopic peripheral pulmonary arteries in all lobes were normal without any features of pulmonary hypertension. Bronchial arteries close to the aneurysms were dilated but were not affected by the arteritis. Examination of the bronchi showed

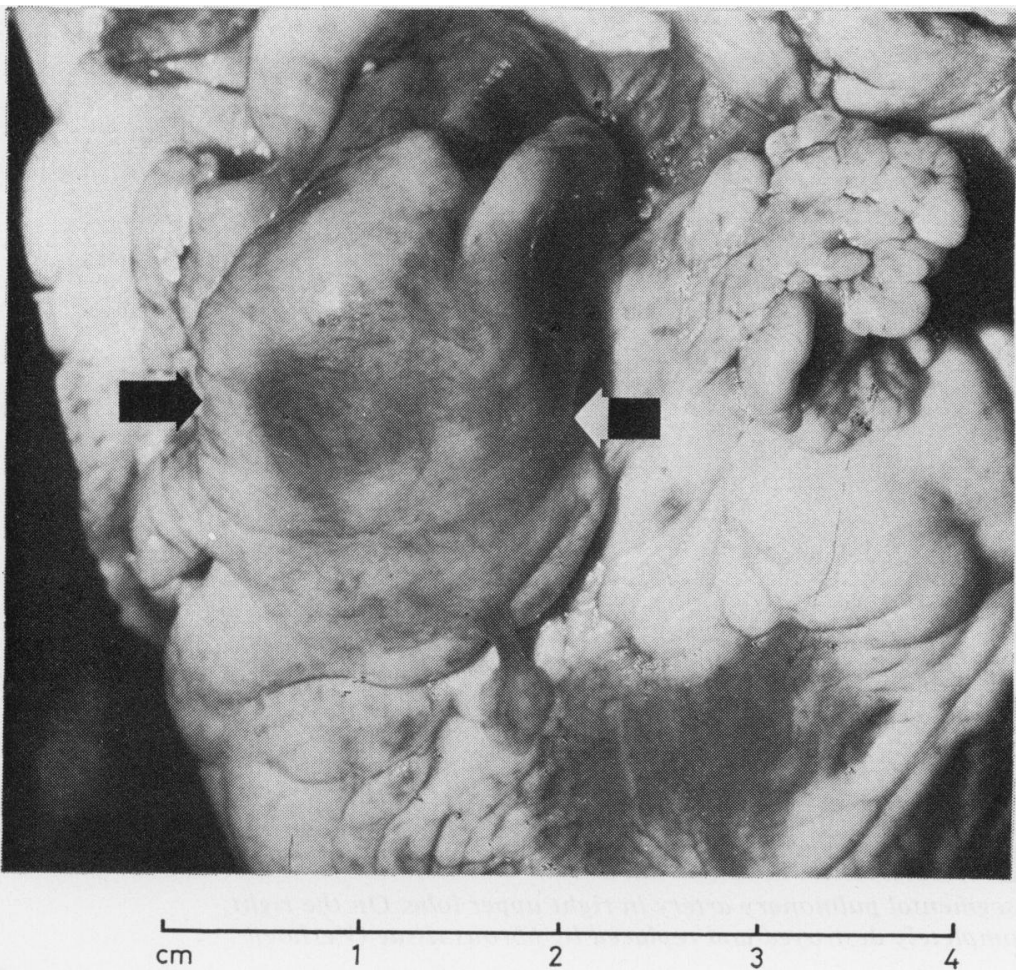

Fig. 3 Anterior surface of heart. Arrows mark the edges of a large left coronary aneurysm. 


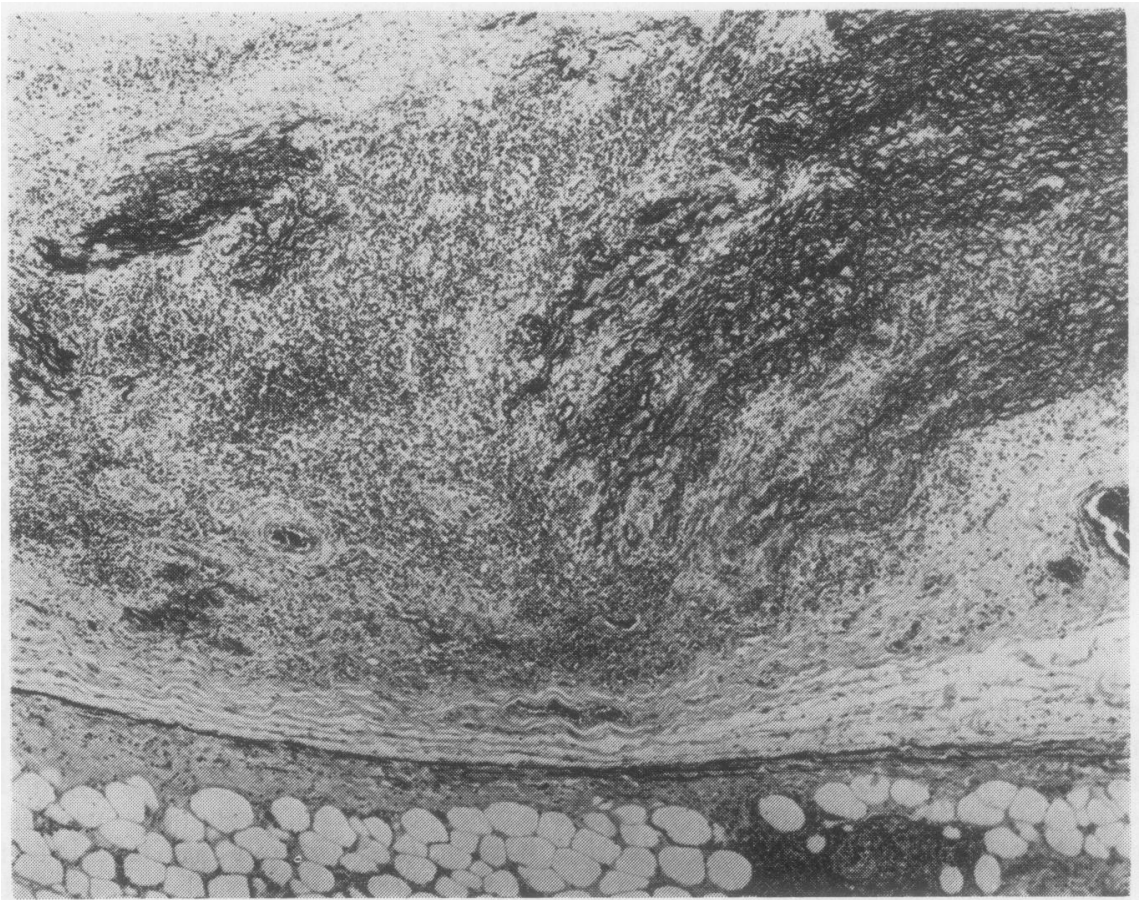

Fig 4 Descending thoracic aorta showing complete destruction of elastic tissuc in segment of media. There is a heavy chronic inflammatory cell infiltrate in the media and periarterial tissues, and in the intima, seen at top of figure on left (Orcein stain for elastic tissue $\times 74$ ).

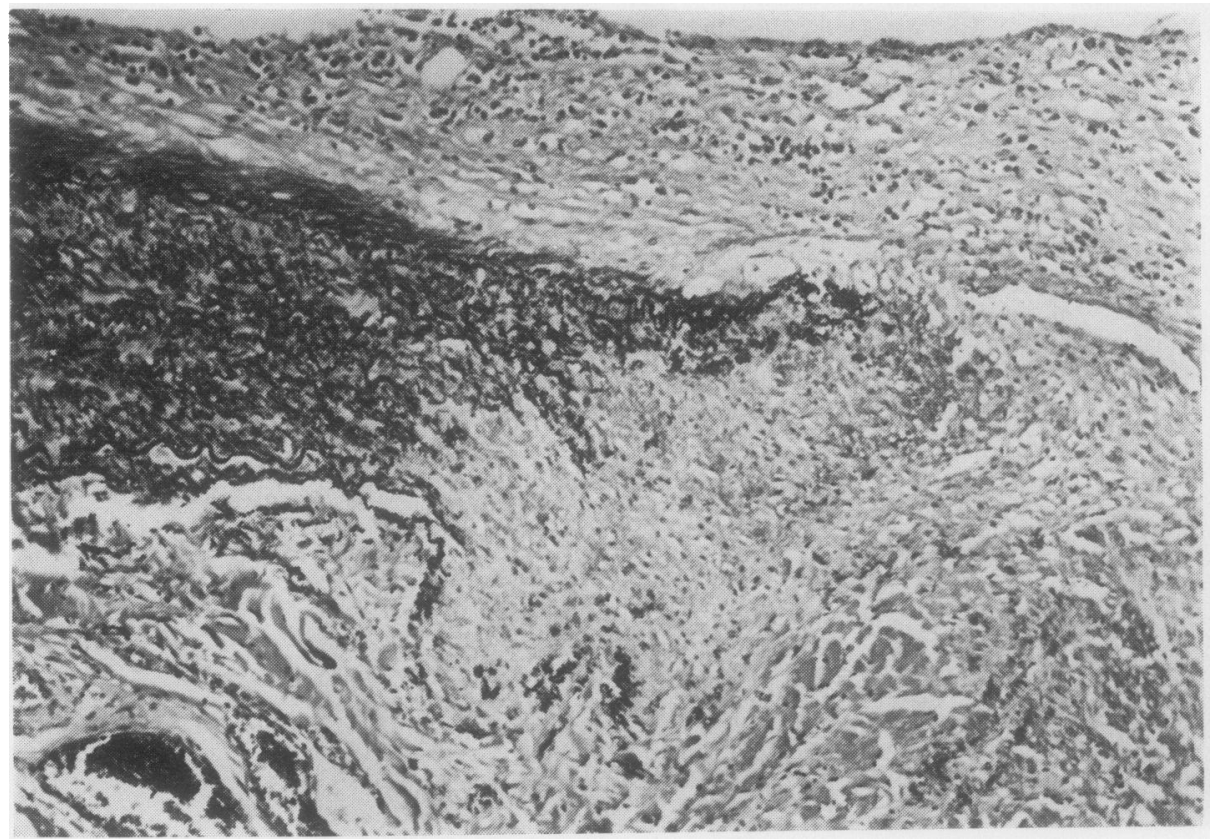

Fig. 5 Aneurysm of a segmental pulmonary artery in right upper lobe. On the right, medial elastic tissue is completely destroyed and replaced by fibrous tissue (Verhoeff Van Gieson $\times 120$ ). 
basement membrane thickening and an increase in smooth muscle and goblet cells, consistent with the history of asthma.

The coronary arteries distal to the aneurysms already described showed fibrous replacement of the wall and severe stenosis, and there was a healed subendocardial infarct in the anterolateral wall of the left ventricle. The endocardium and pericardium had a slight focal lymphocytic infiltrate, but the valves were normal, and there were no changes of chronic rheumatic heart disease.

There was evidence of arteritis affecting a few microscopic vessels. A small hepatic artery showed replacement of the media by fibrous tissue. On reviewing the pathology of the spleen a microscopic trabecular artery was found to show fragmentation of the internal elastic lamina and destruction of the media with an infiltrate of chronic inflammatory cells. There was pronounced intimal proliferation (fig 6). None of the small vessels showed features of acute arteritis such as polymorphonuclear leucocyte inflltration or fibrinoid necrosis.

The other organs showed minor changes only, and the genital tract, brain, meninges, and cerebral vessels were all normal.

\section{Discussion}

Aneurysms of the pulmonary artery are one of the rarest lesions of the cardiovascular system (Fairbairn et al, 1972). This perhaps explains the delay in finding the correct cause of the radiological appearances in this patient, even in the presence of systemic arteritis. Treatment did not appear to alter the course of the disease, the final catastrophe being aortic dissection. The cleft in the aortic wall may have been initiated by haemorrhage in a highly vascular damaged area of the media, with subsequent rupture into the aortic lumen.

The principal conditions associated with arteritis

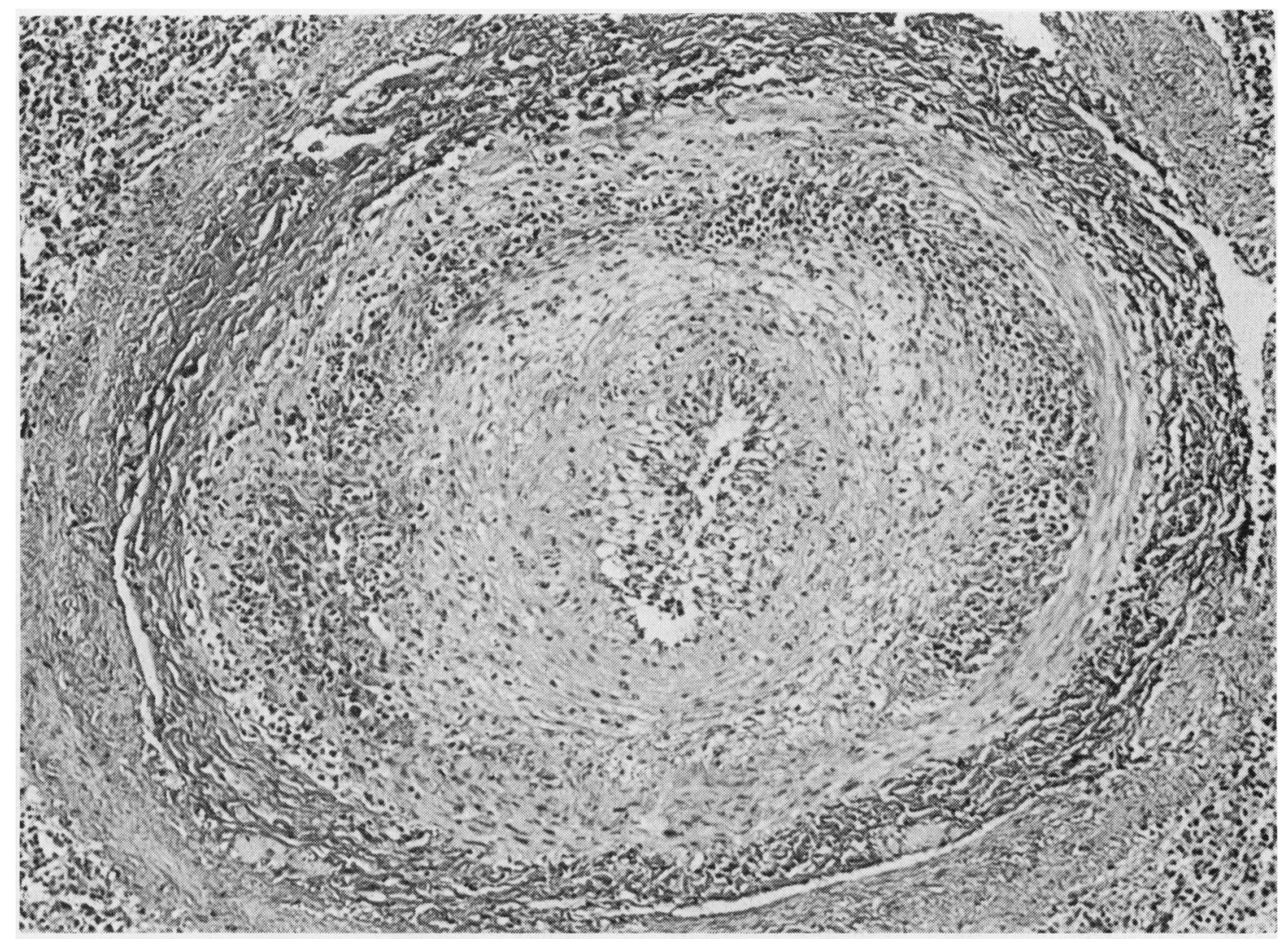

Fig 6 A trabecular artery in the spleen showing marked intimal thickening and a chronic inflammatory cell infiltrate in the media and intima, around the internal elastic lamina (Haematoxylin and eosin $\times 80)$. 
of large vessels, including the aorta, are syphilis, ankylosing spondylitis, rheumatoid disease, rheumatic fever, Reiter's syndrome, Takayasu's disease, and giant-cell arteritis (Hudson, 1965; Marquis et al, 1968). Syphilis is unlikely to be the cause of this arteritis. Syphilitic serology was negative, there was no aneurysm of the ascending aorta, coronary ostial stenosis, or aortic valve disease, and there were no other clinical or neurological features to suggest the condition. The histological features were similar to syphilis, but these may be found in other forms of arteritis (see below). The rheumatic-rheumatoid group of conditions is excluded in this case by the absence of relevant clinical features and the normal heart valves.

Takayasu's disease is a form of arteritis of unknown aetiology, but it occurs principally in young women (Lupi et al, 1977). Athough the pulmonary arteries may be affected (Nasu, 1975; Lupi et al, 1975 ) the consequence is stenosis and not aneurysm formation, following the same pattern as the systemic disease. Finally, the aorta or its major branches may be affected in giant-cell arteritis (Klein et al, 1975), but aneurysm formation is rare. Our patient was rather young for this condition, and the histological findings, particularly the absence of giant cells, excludes the diagnosis.

There remain a few cases in which aortitis is found without any known cause (Heggtveit et al, 1963; Marquis et al, 1968). Pomerance et al (1977), reviewing 63 cases of aortic aneurysm, found seven such cases. The histological appearances include granulomatous infarct-like lesions or changes indistinguishable from syphilis, and this latter appearance is similar to our own case.

Aortic dissection is usually due to cystic medionecrosis, which may occur in association with the Marfan syndrome. While dissection is uncommon in syphilis (Hudson, 1965), there have been several reports of its occurrence in giant-cell arteritis (Magarey, 1950; Harris, 1968; Klein et al, 1975). This patient had no family history or skeletal evidence of Marfan syndrome and no evidence of cystic medionecrosis. The dissection was presumably related solely to the arteritis.

Most aneurysms of the pulmonary arteries are not associated with arteritis but occur in the extrapulmonary vessels in association with congenital cardiovascular abnormalities (Boyd and McGavack, 1939; Deterling and Clagett, 1947). They have also been described in chronic pulmonary hypertension (Spencer, 1977), which was not present in our patient. The types of pulmonary artery aneurysm that have been recognised in association with arteritis are syphilitic aneurysms, mycotic aneurysms, and aneurysms associated with peripheral venous thrombosis (Hughes-Stovin $\overrightarrow{0}$ syndrome). Syphilis only rarely affects the pul- $\frac{\partial}{\sigma}$ monary arteries, and the main pulmonary arteries $\frac{\bar{\rho}}{\sigma}$ are chiefly affected (Boyd and McGavack, 1939). Our reasons for rejecting this diagnosis have been given above.

Myotic aneurysms due to infected emboli from endocarditis or endarteritis are the most common $\overrightarrow{\vec{\omega}}$ aneurysms in the smaller pulmonary arteries $\stackrel{\omega}{\omega}$ (Charlton and DuPlessis, 1961; Gorodezky et al, $\overrightarrow{\hat{x}}$ 1974), but in this man there was no suspicion of $\underset{\omega}{\omega}$ endocarditis during life, and at necropsy no source $\omega$ of infected emboli was found. Several cases of pulmonary artery aneurysm associated with per- $\stackrel{\oplus}{\omega}$ ipheral venous and sagittal sinus thrombosis have 을 been described (Hughes and Stovin, 1959; Kirk and Seal, 1964). The aetiology is obscure, but no $\rightarrow$ evidence of venous disease was found in ourc patient.

Some clinical features of this case are similar to polyarteritis nodosa. In the series of Rose and Spencer (1957) 12 of 32 patients with polyarteritis and lung involvement had asthma, preceding the onset of arteritis by up to seven years. Our patient developed asthma five years before his arteritis became apparent. He had no peripheral blood

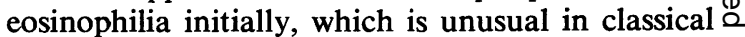
polyarteritis, but he developed a mild eosinophilia $\overrightarrow{\overrightarrow{0}}$ five years later. The histological findings indicate 3 that the arteritis had been present for several years, and we think it likely that his asthma was? part of the same disease process. Histologically, however, the arteritis was unlike polyarteritis nodosa, which affects small and medium-sized $\times$ arteries rather than large vessels, and in which $\frac{3}{3}$ fibrinoid necrosis and polymorphonuclear leucocyte infiltration are characteristic features. In 0 addition, no lesions were seen in the intrarenal arteries, arterioles, or glomeruli. Pulmonary artery윽 aneurysms are not a feature of polyarteritis nodosa.

Until the underlying processes, which are prob-N ably immunological, are better understood, the classification of most cases of arteritis remains 0 confused and is based only on clinical patterns of $\tilde{\omega}$ disease. We have discussed the recognised causes of arteritis and aneurysm formation and considere that our case is unique. This patient had wide- $\mathbb{\Phi}$ spread arteritis of unknown aetiology affecting all ${ }^{\stackrel{\rho}{+}}$ three layers of the vessel wall and a few micro- 0 scopic arteries in addition to many large and $\stackrel{\vec{D}}{\circ}$ medium-sized vessels. The arteritis resulted in extensive aneurysm formation in both systemic $\stackrel{\mathbb{Q}}{\varnothing}$ and pulmonary vessels.

We thank Dr R M E Seal for his helpful commentso 
on the pathological findings and Mr P M Langham for the photomicrographs.

\section{References}

Boyd, L J, and McGavack, T H (1939). Aneurysm of the pulmonary artery. A review of the literature and report of two new cases. American Heart Journal, 18, 562-578.

Charlton, R W, and DuPlessis, L A (1961). Multiple pulmonary artery aneurysms. Thorax, 16, 364-371.

Deterling, R A, and Clagett, O T (1947). Aneurysm of the pulmonary artery; review of the literature and report of a case. American Heart Journal, 34, 471-499.

Fairbairn, J F, Juergens, J L, and Spittell, J A (1972). Peripheral Vascular Diseases, 4th edition. W B Saunders, Philadelphia.

Gorodezky, M, Maroto, J M, Mota, J, Cardenas, M, and Contreras, R (1974). Mycotic aneurysms of the pulmonary artery. Chest, 66, 214-216.

Harris, M (1968). Dissecting aneurysm of the aorta due to giant-cell arteritis. British Heart Journal, 30, 840-844.

Heggtveit, H A, Hennigar, G R, and Morrione, T G (1963). Panaortitis. American Journal of Pathology, 42, 151-172.

Hudson, R E B (1965). Cardiovascular Pathology, volume 1. E Arnold, London.

Hughes, J P, and Stovin, P G I (1959). Segmental pulmonary artery aneurysms with peripheral venous thrombosis. British Journal of Diseases of the Chest, $53,19-27$.

Kirk, G M, and Seal, R M E (1964). False aneurysm of the pulmonary artery with peripheral venous thrombosis. Thorax, 19, 449-453.
Klein, R G, Hunder, G G, Stanson, A W, and Sheps, $S$ G (1975). Large artery involvement in giant-cell (temporal) arteritis. Annals of Internal Medicine, 83, 806-812.

Lupi, H E, Sanchez, T G, Horwitz, S, and Gutierrez, E (1975). Pulmonary artery involvement in Takayasu's arteritis. Chest, 67, 69-74.

Lupi, H E, Sanchez, T G, Marcushamer, J, Mispireta, J, Horwitz, S, and Vela, J E (1977). Takayasu's arteritis. Clinical study of 107 cases. American Heart Journal, 93, 94-103.

Magarey, F R (1950). Dissecting aneurysm due to giant-cell aortitis. Journal of Pathology and Bacteriology, 62, 445-446.

Marquis, Y, Richardson, J B, Ritchie, A C, and Wigle, E D (1968). Idiopathic medial aortopathy and arteriopathy. American Journal of Medicine, 44, 939-954.

Nasu, T (1975). Takayasu's truncoarteritis in Japan. Pathologia et Microbiologia, 43, 140-146.

Pomerance, A, Yacoub, M H, and Gula, G (1977). The surgical pathology of thoracic aortic aneurysms. Histopathology, 1, 257-276.

Rose, G A, and Spencer, H (1957). Polyarteritis nodosa. Quarterly Journal of Medicine, new series, 26, 43-81.

Sanerkin, N G, and Evans, D M D (1965). The sputum in bronchial asthma: pathognomonic patterns. Journal of Pathology and Bacteriology, 89, 535-541.

Spencer, H (1977). Pathology of the Lung, 3rd edition, volume 2. Pergamon Press, Oxford.

Requests for reprints to: Dr J P R Hartley, Asthma Research Unit, Sully Hospital, Penarth, South Glamorgan CF6 2YA. 